\title{
The interplay between expressed parental anxiety and infant behavioural inhibition predicts infant avoidance in a social referencing paradigm
}

\author{
Evin Aktar, Mirjana Majdandžić, Wieke de Vente, and Susan M. Bögels \\ Research Institute of Child Development and Education, Cognitive Science Center Amsterdam, University of \\ Amsterdam, Amsterdam, The Netherlands
}

\begin{abstract}
Background: Anxiety aggregates in families. Environmental factors, such as modelling of anxious behaviours, are assumed to play a causal role in the development of child anxiety. We investigated the predictive value of paternal and maternal anxiety (lifetime anxiety disorders and expressed parental anxiety) on infants' fear and avoidance during encounters with social and nonsocial novel stimuli in a social referencing (SR) paradigm. Methods: A total of 122 12-month-old infants participated in this study separately with their fathers and mothers (parents with lifetime: social anxiety disorders [ $n=47]$, other types of anxiety disorders [ $n=33$ ], comorbid social and other types of anxiety disorders $[n=52]$ and without anxiety disorders $[n=112])$. Infants were confronted with a stranger and a mechanical dinosaur as novel stimuli in two SR situations. Infants' avoidance as well as fear and parents' expressed anxiety were observed. Infants' behavioural inhibition (BI) was separately observed in structured tasks. Results: Parental lifetime anxiety disorders did not significantly predict infant fear or avoidance. Expressed parental anxiety interacted with BI to significantly predict infant avoidance, revealing a positive association between expressed parental anxiety and infant avoidance among infants with moderate-to-high BI. The association between infant avoidance and expressed parental anxiety was not significantly different for mothers and fathers, pointing to an equally important role of fathers at this young age. Infant fear was significantly predicted by infant BI, but not by expressed parental anxiety. Conclusions: Infants with a temperamental disposition for anxiety (BI) may learn from both paternal and maternal anxious signals and become avoidant towards novelty when their parents express anxiety. This link between expressed parental anxiety and infant avoidance for moderate-to-high BI children, that seems to hold across contexts and to be independent of lifetime parental anxiety disorders, may be a mechanism explaining early intergenerational transmission of anxiety. Keywords: Social referencing, parental anxiety disorders, expressed parental anxiety, behavioural inhibition.
\end{abstract}

\begin{abstract}
Introduction
Anxiety disorders are one of the most prevalent psychiatric conditions in adults (Kessler, Chiu, Demler, \& Walters, 2005) and children (Kashani \& Orvaschel, 1990). Evidence supports the view that anxiety disorders in parents contribute to the development of anxiety in children. In addition to moderate and nonspecific genetic influences (Eley, 2001; Hettema, Neale, \& Kendler, 2001), environmental influences seem to be more pronounced for the intergenerational transmission of anxiety (Bögels \& Brechman-Toussaint, 2006; Murray, Creswell, \& Cooper, 2009). Anxious parents may contribute to the development of childhood anxiety via anxious modelling and a lack of positive reinforcement on approaching unfamiliar or potentially threatening situations (i.e. encouragement; Fisak \& Grills-Taquechel, 2007; Murray et al., 2009).
\end{abstract}

Evidence of early environmental influences of parental anxiety relies on the infants' emerging social referencing (SR) skills towards the end of the first year (Carpenter, Nagell, Tomasello, Butterworth, $\&$ Moore, 1998). SR occurs when infants make use of the adults' emotional signals to determine how to act when confronted with unfamiliar people, objects or situations (Feinman, 1982). SR may be a mechanism contributing to the early learning of fear from parents via observational learning and modelling: infants of anxious parents are more likely to be provided with expressions of parental anxiety (Muris, Steerneman, Merckelbach, \& Meesters, 1996) during SR and may model anxious coping strategies (e.g. avoidance) from their parents (Fisak \& Grills-Taquechel, 2007; Murray et al., 2009, Rapee, 2001).

Infant characteristics may also influence SR. Behavioural inhibition (BI), a biologically driven avoidant, fearful and withdrawn attitude towards novelty (Fox, Henderson, Marshall, Nichols, \& 
Ghera, 2005; Kagan \& Snidman, 1999; Rubin, Coplan, \& Bowker, 2009), and a temperamental predisposition for childhood anxiety, has been found to moderate the effects of parental anxiety on infants' avoidance during SR situations (Murray et al., 2008). Infants with high BI are at risk for developing anxiety disorders (Biederman, Rosenbaum, Chaloff, \& Kagan, 1995; Rosenbaum et al., 1993), especially of the social type (Rubin et al., 2009). Studies have reported increased levels of BI in children of anxious parents (Rosenbaum et al., 1993).

The interplay between BI and modelling of parental anxiety via SR may play an important role in the development of child anxiety disorders. As suggested by diathesis-stress (Zuckerman, 1999) and vulnerability-stress models (Ingram \& Luxton, 2005; Nigg, 2006), the temperamental predisposition of BI may constitute vulnerability for the effects of adverse rearing environments. The 'differential susceptibility to environmental influences hypothesis' (Belsky \& Pluess, 2009) is a recent extension of these models on the effects of positive rearing: highly inhibited children are not only assumed to be more susceptible to adverse rearing environments, but also to benefit more from adaptive rearing environments, making them more open to the effects of the environment and parenting 'for better and for worse' (Belsky \& Pluess, 2009). All three models predict that high $\mathrm{BI}$ children would be the highest risk group with respect to the effects of anxious parenting via SR. The differential susceptibility to environmental influences hypothesis further predicts that high BI children would benefit the most from encouraging parenting.

To investigate the effects of anxious maternal reactions on child behaviour via SR in an experimental design, mothers' behaviour towards novel social and nonsocial stimuli has been manipulated either through direct instructions, or by training anxious responses (Bradshaw, Goldsmith, \& Campos, 1987; Gerull \& Rapee, 2002; De Rosnay, Cooper, Tsigaras, \& Murray, 2006; Dubi, Rapee, Emerton, \& Schniering, 2008). Results from these studies provide support for a link between parental anxiety and child fear/ avoidance: even a relatively brief negative (fear or disgust) reaction (range: $30 \mathrm{~s}$ to $1 \mathrm{~min}$.) from the mother may be enough for the toddlers to become fearful and avoidant towards a novel toy (Gerull \& Rapee, 2002). The SR processes have also been experimentally investigated in response to strangers (De Rosnay et al., 2006), and the results provide evidence for the vulnerability of high BI infants to the negative influence of anxious maternal reactions.

Only one study has investigated SR processes with clinically anxious mothers. Murray et al. (2008) examined the transmission of anxiety among mothers with $(n=79)$ and without $(n=77)$ social anxiety disorder at 10 and 14 months to investigate whether the coemergence of SR and stranger anxiety (Sroufe, 1977) during late infancy makes infants vulnerable to parental social anxiety. This SR paradigm allowed the infant to observe the mother in interaction with a stranger who subsequently approached the infant and interacted with him/her. The paradigm enabled studying the link between maternal anxiety and simultaneous and subsequent infant fear/avoidance towards the stranger. Socially anxious mothers showed more anxiety than reference mothers at both assessments. Murray et al. (2008) also observed a longitudinal change with high BI children of socially anxious mothers becoming more avoidant from 10 to 14 months, supporting the central assumption of vulnerability-stress models. Furthermore, the interplay between parental social anxiety disorder and infant BI was accounted for by lower levels of encouragement in mothers with social anxiety disorders.

Murray et al. (2008) provided the first evidence of early transmission of anxiety with clinically anxious mothers via SR. Nevertheless, several aspects of this transmission await further investigation. First, information on paternal effects is absent. Recent models on the development of anxiety (Bögels \& Perotti, 2011; Bögels \& Phares, 2008) assign fathers an essential role in the development of anxiety based on their greater evolutionary experience in exploring the external world. Therefore, the inclusion of fathers with and without anxiety disorders on infant SR studies is needed. Second, it remains unknown whether the transmission only occurs from socially anxious mothers or also from mothers without anxiety disorders mothers or mothers with other types of anxiety disorders. Third, it remains unclear whether the transmission occurs only in contexts that provoke social anxiety, or whether it also applies to other, nonsocial contexts.

In this study, we observed 12-month-old infants' responses to novelty in SR situations. First, we aimed to extend the current knowledge of SR processes by exploring the fathers' role in the intergenerational transmission of anxiety via SR. We expected that fathers would be as important as mothers in the transmission of anxiety. On the basis of previous evidence with mothers, infants were expected to show more fear/avoidance if they were highly behaviourally inhibited, if their parents had a lifetime anxiety disorder, or if their parents had high levels of expressed anxiety during the task. Second, in light of the differential susceptibility hypothesis, a larger negative influence of expressed parental anxiety and a larger positive influence of parental encouragement on infant fear/avoidance was expected for high BI infants in both SR situations. Third, to explore diagnosis-dependency and anxiety subtype specificity in the transmission of anxiety, we included infants of parents with lifetime social anxiety disorder, with other types of anxiety disorders, with comorbid social and other types of anxiety disorders and parents without anxiety disorders. If parental expressions of anxiety are diagnosis- 
dependent, one would expect higher levels of expressed anxiety among parents with lifetime anxiety disorders (vs. parents without lifetime anxiety disorders). If parental expressions of anxiety are diagnosis-specific, then one would expect that the link between lifetime anxiety disorders and expressed parental anxiety would differ as a function of the type of parental lifetime anxiety disorders. Similarly, if the transmission of anxiety is diagnosisdependent, one would expect higher levels of fear/ avoidance among infants of parents with (vs. without) lifetime anxiety disorders. If the transmission of anxiety is diagnosis-specific, then one would expect that the link between lifetime anxiety disorders and the infant fear/avoidance would differ as a function of the type of parental lifetime anxiety disorders. Fourth, to explore context-specificity in the transmission of anxiety, we observed SR processes in response to a novel social (a stranger) or nonsocial (a remote control dinosaur) stimulus. If parental expressions of anxiety are context-specific, then one would expect that the link between lifetime anxiety disorders and expressed parental anxiety would differ between the social and nonsocial SR situations. Similarly, if the transmission of anxiety is context-specific, then one would expect that the link between expressed parental anxiety and infant fear/ avoidance would be different in social versus nonsocial SR situations.

\section{Methods}

\section{Participants}

The sample consisted of 122 couples with their 12month-old first-born infant. The families are part of a larger group of participants recruited from the general population for an ongoing longitudinal study on social development from infancy to middle childhood which includes a prenatal measurement (see below) and a 4month measurement (not used in this study). Couples expecting their first child were recruited via advertise- ments in magazines and on parenting websites, or via flyers provided by midwives, pregnancy courses and baby shops. The project was approved by the ethics committee of University of Amsterdam. Participants provided written informed consent prior to their participation.

The SR tasks were completed by 120 mothers and 122 fathers. Age and sociodemographic characteristics of the sample are presented in Table 1 .

\section{Materials and procedure}

Each parent visited the lab separately when his/her infant was 12 -months old: $54 \%$ of the infants visited with their mother first, and $46 \%$ with their father. The order of the parental visits was included in the analyses as a control variable. Measurements of infant BI and SR were conducted and recorded during these visits (three additional BI tasks were conducted during a home visit). BI tasks were conducted before the SR tasks during the visits.

Parental anxiety status Each parent's anxiety status was obtained via the anxiety disorder interview schedule (ADIS; Di Nardo, Brown, \& Barlow, 1994). The ADIS is a semistructured clinical interview based on DSM-IV criteria and was conducted at the prenatal measurement by four trained and experienced interviewers (psychology/educational sciences graduates) to assess parent's current and lifetime anxiety disorder status. Agreement about difficult cases was obtained by post hoc consultation of an expert in anxiety disorders (SB). To establish interobserver reliability, a trained psychologist recoded $10 \%$ of the interviews that were videotaped in the prenatal measurement. The percentage interobserver agreements for all ADIS diagnoses were based on the presence or absence of the specific anxiety disorder. The percentage interobserver agreements ranged from $90 \%$ to $100 \%$ per anxiety disorder with a mean of $97.55 \%(S D=2.95)$.

As our theoretical interest was on the predispositions of parental anxiety, rather than expressed parental anxiety (that was separately observed during the SR tasks), we assigned parents to the following parental

Table 1 Sociodemographic characteristics of the sample

\begin{tabular}{|c|c|c|c|c|c|}
\hline & & Mothers & Fathers & Girls & Boys \\
\hline $\mathrm{N}$ & & 122 & 122 & 67 & 55 \\
\hline Age $M(S D)$ & & $31.78(4.20)$ & $34.64(5.33)$ & $1.03(0.06)$ & $1.00(0.04)$ \\
\hline Dutch origin (\%) & & 90.98 & 95.08 & & \\
\hline \multirow[t]{2}{*}{ Dutch parental origin (\%) } & $\mathrm{M}$ & 90.16 & 94.26 & & \\
\hline & $\mathrm{F}$ & 84.43 & 93.44 & & \\
\hline Educational level $^{\mathrm{a}} M(S D)$ & & $7.06(1.13)$ & $6.62(1.53)$ & & \\
\hline Professional level $^{\mathrm{b}} M(S D)$ & & $8.73(2.09)$ & $8.17(2.66)$ & & \\
\hline \multirow{3}{*}{ Current working status (\%) } & HK & 3.4 & 1.8 & & \\
\hline & PT & 77.6 & 26.3 & & \\
\hline & FT & 10.3 & 67.5 & & \\
\hline Monthly income $^{\mathrm{c}} M(S D)$ & & $4.11(1.41)$ & $4.61(1.33)$ & & \\
\hline
\end{tabular}

M, mother; F, father; HK, housekeeper; PT, parttime; FT, fulltime.

${ }^{\text {aP }}$ arental educational level was assessed using an 8-point scale ranging from 1 (primary education) to 8 (university).

${ }^{\mathrm{b}}$ Parental professional level was assessed using an 11-point scale ranging from 1 (manual labour for which no education is required) to 11 (labour for which a university degree is required).

${ }^{\mathrm{c}}$ Parental income was assessed using a 7 -point scale from 1 ( $<500$ euros/month) to 7 (more than 5,000 euros $\left./ \mathrm{month}\right)$. 
Table 2 Prevalence of lifetime anxiety diagnoses in the parent sample

\begin{tabular}{|c|c|c|c|c|c|c|}
\hline & & \multicolumn{5}{|c|}{ Mother } \\
\hline & & $\mathrm{SA}$ & $\mathrm{OA}$ & $\mathrm{SA} \& \mathrm{OA}$ & None & TOTAL \\
\hline \multirow[t]{5}{*}{ Father } & $\mathrm{SA}$ & 5 & 5 & 7 & 7 & 24 (16) \\
\hline & $\mathrm{OA}$ & 0 & 5 & 3 & 4 & $12(7)$ \\
\hline & SA\&OA & 2 & 4 & 11 & 4 & $21(8)$ \\
\hline & None & 16 & 7 & 10 & 32 & 65 \\
\hline & TOTAL & 23 (14) & 21 (16) & 31 (15) & 47 & 122 \\
\hline
\end{tabular}

SA, Social anxiety disorder; OA, other types of anxiety disorders; SA \& OA, social anxiety disorder and at least one other anxiety disorder; None, no anxiety disorder.

Parental anxiety disorder groups were formed on the basis of current and/or past anxiety disorders. Information is provided on mothers and fathers separately as totals and on how their disorders relate to each other as couples (parents of the same infant). For example, there are seven mothers without anxiety diagnosis who have a partner with social anxiety disorder. The numbers in parentheses indicate the number of parents only with the current anxiety disorders.

anxiety groups based on the current and/or lifetime anxiety diagnoses as measured in the prenatal interview: 'social anxiety disorder', 'other types of anxiety disorders', 'comorbid social and other type(s) of anxiety disorders' and 'no anxiety disorder' groups. None of the mothers were in treatment, whereas one father in the comorbid group was in treatment for anxiety problems. The sample sizes for each group are summarized in Table 2.

\section{SR tasks.}

Stranger SR task. In phase I, a female stranger entered the room and engaged the parent in a 2 -min conversation about his/her experiences of parenthood, while the infant was watching the interaction from a high chair. In phase II, the stranger told the parent that she would pick up the infant and asked the parent to inform the infant. In phase III, the stranger gradually approached the infant, picked him/her up, lifted him/her in the air and put him/her on the floor (for more information, see Murray et al., 2008). The strangers maintained a neutral attitude during the SR task. Different strangers were used for the mother and father visits.

Dinosaur SR task. A remote control dinosaur was placed two metres from the infant, and displayed a pattern of movements and noises. In phase I, parents remained neutral. In phase II, parents were instructed to talk about the dinosaur. In phase III, parents actively encouraged the infant to approach the dinosaur. Two different dinosaurs were used and counterbalanced between the mother and father visits. The order of tasks was fixed for each visit: first stranger, then dinosaur.

Murray et al.'s (2008) coding protocol was used to code parental and infant behaviour in the stranger SR task. We developed a parallel coding scheme for the dinosaur SR task. All behavioural dimensions from parents and infants (see below) were rated on each time interval (coding units within a phase) as a single rating. The ratings were made on a 5 -point scale unless stated otherwise.

\section{Parent behaviour during SR tasks.}

1. Expressed parental anxiety during SR tasks was based on facial (e.g. anxious, frozen faces), bodily (e.g. fidgeting, rigid posture) and verbal expressions of anxiety.
2. Parental encouragement involved encouragement of infant positive engagement with the stranger and of approach to the dinosaur through smiles and looks, or simple comments. This behaviour was rated on a 3-point scale.

3. Parental overcontrol involved parental attempts to control or intervene during SR situation (e.g. gesturing to the infant and signalling for the infant's attention when the infant has not sought attention, or making specific attributions on behalf of the infant).

\section{Infant behaviour during SR tasks.}

1. Infant fear was based on facial (e.g. wide eyes, cry face), bodily (e.g. sunken-in posture, decrease in activity) and vocal (e.g. whining, crying) expressions of fear.

2. Infant avoidance involved behaviours like looking/ turning away, hiding behind the parent, or ignoring the stimulus.

3. Infant approach to the stranger involved positive engagement: smiling, laughing to the stranger, positive vocalizations, and approach to the dinosaur: active attempts to approach or touch the dinosaur.

4. Infant baseline negativity was based on facial, bodily and vocal expressions of negative emotional tone. It was rated in the last minute before the task started and used as a control variable, as infants varied in their initial negativity before the SR tasks.

5. Infant's looks at the parent were a count of the number of times the infant looked at the parent during SR situations. Looks at the parent are indicative of active information seeking and serve as a preliminary measure of the extent to which social referencing occurs during the SR tasks.

For the coding of infant and parent behaviour, each phase was divided into $1 \mathrm{~min}$ or $30-\mathrm{s}$ time intervals. In the stranger SR task, the time intervals in the second and third phases were determined by a given action of the stranger. Final scores in each dimension were obtained by averaging the interval scores. Two pairs of observers were trained to code either infant or parent behaviour during SR. Observers were blind to parents' diagnostic status and infants' BI. To prevent potential carryover effects stemming from the observation of mother-infant and father-infant dyads of the same 
family, each observer in both pairs coded either the father (or the infant with father) or the mother (or the infant with mother) of a family except for $20 \%$ of the SR data that was double-coded for interobserver reliability. The final scores for the double-coded data were obtained by averaging the ratings of the two observers.

The interobserver reliabilities for the parent and infant variables (intraclass correlations, ICC) were .78 for parental anxiety, .77 for parental encouragement, .90 for infant fear, .65 for infant avoidance, .75 for infant approach, .78 for infant baseline negativity and .94 for looks at the parent. Parental overcontrol was not reliably coded and was only observed in $4.19 \%$ of the measurements. This variable was not considered further.

$B I$ tasks Infant BI was measured via 11 social and nonsocial tasks from several well-known standard BI laboratory instruments. Three tasks were from the Laboratory Temperament Assessment Battery (LABTAB; Goldsmith \& Rothbart, 1996): stranger approach (a male stranger approached and picked up the infant), unpredictable mechanical toy (a large, remote control toy train drove across the table) and masks (the experimenter appeared from behind a curtain successively showing three masks). Three unpredictable mechanical toy tasks were modelled after Rothbart (1988): buzzing animal (a small vibrating animal toy was placed within arm's reach distance of the infant), ambulance (a toy ambulance with light and sound rode towards the infant) and horse (a neighing toy horse approached the infant). Four discomfort tasks developed by Kochanska, Coy, Tjebkes, and Husarek (1998) were used: ice (an ice cube was held against the foot and the neck of the infant), lemon (the infant was given two spoons of diluted lemon juice), spray (water was sprayed on the infant's face) and blender (the infant was exposed to the noise of a blender). Finally, the truck task (Calkins, Fox, \& Marshall, 1996; Fox, Henderson, Rubin, Calkins, \& Schmidt, 2001) was used: a female stranger came into the room, played with a toy truck with blocks and invited the infant to join.

The BI tasks of unpredictable mechanical toy, stranger approach and masks were administered in the mother's visit, whereas the truck and the four discomfort tasks were conducted in the father's visit. Buzzing animal, ambulance and horse tasks were conducted during a home visit with both parents present. Parents sat behind the infant and remained neutral during the BI tasks. The order of the BI tasks was fixed for all infants.

For each BI task, several indices of infant behaviour were coded, such as facial fear, bodily fear, escape, distress vocalizations, the latency to touch the toy, the latency of the first fear reaction, startle response and proximity to parent (see Goldsmith \& Rothbart, 1996).

The scores were first averaged across epochs (coding intervals), then standardized and averaged for each BI task, and then aggregated into a single BI score. Six observers were trained by a master coder (MM) to code the $11 \mathrm{BI}$ tasks. To establish interobserver reliability, the master coder coded $20 \%$ of each observer's data pool. Average interobserver reliability of coded variables was .83 (ICCs ranged from .60 to .93, [SD =.11] per task, and from .39 to $.99[S D=.16]$ per variable).
Among 19 observed variables, two showed little variance and were not reliably coded: The first variable concerned the extent to which infants talked to the stranger in the truck task (ICC $=.39 ; 79.68 \%$ of the infants did not talk). The second variable was the startle response in the spray task (ICC $=.56 ; 77.47 \%$ of the infants showed a startle response). The internal consistency of the total score across the 11 BI tasks was .79.

\section{Statistical analyses}

The design consisted of two SR tasks (stranger and dinosaur) that were repeated with both parents, giving rise to a hierarchical data structure with four observations per infant. A two-level regression model (consisting of task and parent's gender) was fitted for each outcome variable (i.e. infant fear, infant avoidance and expressed parental anxiety). The significance of the effects was evaluated at $p<.05$. Scores on continuous outcome and predictor variables were standardized. For each analysis, the levels task and parent gender, along with other predictors, and the intercept were analysed using a fixed-effects model. Parental anxiety disorders were dummy coded with parents without anxiety disorders as the reference group. Infant $\mathrm{BI}$, expressed parental anxiety, parental encouragement and looks at the parent were entered as continuous variables in the models. Inspection of distributions indicated sufficient normality for all variables except infant baseline negativity. As infants were neutral in the majority of the cases, this variable was categorized $(0$ : neutral, 1 : negative). To check the assumptions of multilevel models, the distributions of residuals were checked for normality and linearity following the main analyses and found to be satisfactory. Maximum likelihood was the estimation method. Multilevel models were analysed using PASW Statistics 18, Release Version 18.0.3. (IBM, Somers, NY, USA).

Infant fear and avoidance were analysed in separate multilevel models. Initially, each model consisted of main effects (of parent gender, SR task, parental lifetime anxiety disorders, expressed parental anxiety during SR, parental encouragement, infant BI, looks at the parent, infant baseline negativity, and order of the visits) only. Subsequently, theoretically relevant interactions were added one by one to the main-effects model. Interaction terms were kept in the model or removed based on likelihood ratio tests and $t$-tests.

For the models of fear and avoidance, we first included the cross-level interaction between infant and parent gender to investigate whether boys' and girls' expressions of fear and avoidance differed between mother and father visits. Second, the cross-level interaction between parent gender and infant BI was included to investigate whether the link between infant BI and fear/avoidance differed across mothers and fathers. Third, we included the cross-level interaction between parent gender and expressed parental anxiety to investigate whether the link between infant fear/ avoidance and expressed parental anxiety differed between mothers and fathers. Fourth, the cross-level interaction between parent gender and parental encouragement was included in the model to investigate whether the link between infant fear/avoidance and 
parental encouragement differed between mothers and fathers. Fifth, we tested the differential susceptibility hypothesis in the transmission of anxiety, by adding the cross-level interactions between expressed parental anxiety and infant BI, and between parental encouragement and infant BI to the main-effects model, to explore how the links of infant fear/avoidance with expressed parental anxiety and with parental encouragement differed as a function of infant BI. Finally, to test the context-specificity in the transmission of anxiety, we included the interaction between expressed parental anxiety and the task in the models.

To investigate the link between parental lifetime anxiety disorders and expressed parental anxiety, we regressed expressed parental anxiety on parental lifetime anxiety disorders in a multilevel model. The interactions of parental lifetime anxiety disorders with the task were entered in subsequent steps to test context-specificity of expressed parental anxiety.

\section{Results}

\section{Preliminary analyses}

In the study by Murray et al. (2008), infant fear and avoidance during the stranger SR situation were treated as separate outcome measures, as there was no significant association between them and different patterns of results emerged with fear versus avoidance. In the current study, we first calculated the raw associations of fear and avoidance for separate SR tasks with each parent (see Table 3). There were moderate associations between them (with the exception of the fathers during the dinosaur SR task), with fear and avoidance sharing approximately $25 \%-30 \%$ of their total variances. Given the findings of Murray et al. (2008), we treated fear and avoidance as two distinct outcome measures despite their positive association. Second, infant approach was negatively associated with both fear and avoidance, but was not considered in this study due to the theoretical priority of negative reactions. Third, to explore the role of looks at the parent as a potential mechanism for the transmission of anxiety, we computed the correlations between infant BI and looks at the parent. There was only a weak positive association between infant BI and looks at the parent in the stranger SR task in the mother visit, indicating that higher BI infants looked more often to their mother in the stranger SR. Fourth, we calculated the raw correlations between expressed parental anxiety and BI to test the overlap between expressed parental anxiety and BI in parent-infant dyads. This correlation seemed to indicate relatively stronger links between expressed maternal anxiety (vs. paternal) and BI, which may be reflecting greater maternal (vs. paternal) sensitivity to infant BI. Fifth, the correlation between expressed parental anxiety and parental encouragement was investigated following the previous findings of Murray et al. (2008) who found that anxious mothers encouraged their infants less. The correlation was only significant for the fathers in the dinosaur SR task.

\section{Main analyses}

The standardized parameter estimates, standard errors (SE) and $p$ values for the fear and avoidance models are shown in Table 4. Although looks at the parent (per interval, $M=1.20, S D=.78$, range: 0.00 4.06) and the interaction between looks at the parent and infant BI, were initially included in the models to investigate the potential mechanisms behind the transmission of anxiety, they did not significantly predict infant fear or avoidance in any of the models. Therefore, they are not further reported in the analyses. Similarly, the interactions between infant and parent gender, between parent gender and BI and between expressed parental anxiety and SR task were included in the main-effects models of fear and avoidance, but they did not reveal any significant associations. Therefore, these interactions are not further reported in the analyses.

Table 3 Raw correlations between infant measures of fear, avoidance and approach, between looks at the parent and infant BI, between expressed parental anxiety and infant BI and between expressed parental anxiety and parental encouragement.

\begin{tabular}{|c|c|c|c|c|}
\hline & \multicolumn{2}{|c|}{ Mother visit } & \multicolumn{2}{|c|}{ Father visit } \\
\hline & SSR & DSR & SSR & DSR \\
\hline$r$ (infant fear, infant avoidance) & .53 & .55 & .24 & .50 \\
\hline$p$ & $<.001$ & $<.001$ & .009 & $<.001$ \\
\hline r(infant approach, infant fear) & -.42 & -.43 & -.42 & -.49 \\
\hline$p$ & $<.001$ & $<.001$ & $<.001$ & $<.001$ \\
\hline$r$ (infant approach, infant avoidance) & -.31 & -.30 & -.34 & -.30 \\
\hline$p$ & .001 & .001 & $<.001$ & .001 \\
\hline$r$ (looks at the parent, infant BI) & .196 & -.129 & .018 & .078 \\
\hline$p$ & .034 & .168 & .846 & .395 \\
\hline$r($ expressed parental anxiety, infant $\mathrm{BI})$ & .26 & .23 & .17 & .11 \\
\hline$p$ & .004 & .012 & .058 & .212 \\
\hline$r$ (expressed parental anxiety, parental encouragement) & -.04 & -.17 & -.11 & -.32 \\
\hline$p$ & .661 & .073 & .250 & $<.001$ \\
\hline
\end{tabular}

SSR, stranger social referencing; DSR, dinosaur social referencing; BI, behavioural inhibition. $p$ values indicate two-tailed significances of the associations. 
Table 4 Parameter estimates for the multilevel models of infant fear and avoidance regressed on parent and infant variables

\begin{tabular}{|c|c|c|c|c|c|c|}
\hline \multirow[b]{2}{*}{ Parameter } & \multicolumn{3}{|c|}{ Fear model } & \multicolumn{3}{|c|}{ Avoidance model } \\
\hline & $\beta$ & $S E$ & $p$ & $\beta$ & $S E$ & $p$ \\
\hline Intercept & -.46 & .10 & $<.001$ & .13 & .13 & .345 \\
\hline Order of the visits ( 0 :first with mother, 1 : first with father) & -.03 & .06 & .654 & -.17 & .08 & .051 \\
\hline Infant baseline negativity ( $0:$ no, 1 :yes) & .48 & .08 & $<.001$ & .15 & .09 & .106 \\
\hline Parent $(0:$ mother, 1 : father) & .06 & .07 & .380 & .02 & .09 & .847 \\
\hline Task (0: SSR, 1: DSR) & .74 & .10 & $<.001$ & -.26 & .10 & .009 \\
\hline Infant Gender & -.15 & .09 & .098 & .06 & .11 & .601 \\
\hline Parent lifetime social anxiety disorder & -.08 & .10 & .436 & -.12 & .13 & .336 \\
\hline Parent lifetime other anxiety disorder(s) & -.03 & .11 & .801 & .09 & .15 & .548 \\
\hline Parent lifetime comorbid anxiety disorder(s) & .08 & .10 & .452 & -.01 & .13 & .973 \\
\hline Expressed parental anxiety & .07 & .04 & .109 & .17 & .07 & .014 \\
\hline Parental encouragement & -.01 & .04 & .728 & -.04 & .06 & .542 \\
\hline Infant BI & .26 & .05 & $<.001$ & .15 & .06 & .007 \\
\hline Parent gender $\times$ expressed parental anxiety & & & & -.11 & .09 & .201 \\
\hline Parent gender $\times$ parental encouragement & & & & .21 & .08 & .014 \\
\hline Expressed parental anxiety $\times$ infant $B I$ & & & & .12 & .05 & .011 \\
\hline
\end{tabular}

SSR, stranger social referencing; DSR, dinosaur social referencing; BI, behavioural inhibition.

For the model with infant fear as the outcome measure, a main-effects only model was first fit $(-2 \log =1145.92, \quad$ AIC $=1189.92, \quad B I C=1281.51)$ The interaction between parent gender and expressed parental anxiety, aiming at exploring differences between parents in the transmission of anxiety, reached marginal significance $(\beta=.13$, $S E=.07, p=.059)$, but failed to significantly improve the fit of the model $(-2 \log =1142.51$, $A I C=1188.51, B I C=1284.27)$. The inclusion of the other theoretically relevant interactions did not reveal any significant effects and did not significantly improve the model. The main-effects model explained $21.62 \%$ of the variance in infant fear.

The final model (see Table 4) revealed that infants showed significantly more fear in the dinosaur SR task $(M=2.30, S D=.98)$ than in the stranger SR task $(M=1.78, S D=.83)$. Infant $\mathrm{BI}$ significantly predicted infant fear during the SR tasks, with increased levels of BI predicting increased fear. Infant fear did not significantly differ between the mother and father visits or between boys and girls. Neither parental anxiety disorders nor expressed parental anxiety significantly predicted infant fear. The association between parental encouragement and infant fear was not significant either.

The fit of the initial main-effects model for infant avoidance was $-2 \log =1260.85, \quad$ AIC $=1304.85$, $B I C=1396.44$. The main-effects model explained $8.59 \%$ of the variance in infant avoidance. Several theoretically relevant interactions (previously described in statistical analyses) were significant and improved this model. First, the inclusion of the interaction between parent gender and expressed parental anxiety was initially significant $(\beta=-.22$, $S E=.08, \quad p=.007)$ and improved the fit $(-2 \log =1253.96, \quad$ AIC $=1299.96, \quad B I C=1395.71)$ indicating a stronger effect for mothers. However, the interaction was no longer significant $(\beta=-.15$,
$S E=.09, p=.098$ ) after including the interaction between parent gender and parental encouragement. Second, the interaction between parent gender and parental encouragement further improved the fit of this model $(-2 \log =1249.89, \quad A I C=1297.89$, $B I C=1397.81$, and indicated a stronger association between infant avoidance and parental encouragement for fathers than for mothers (the association was in the opposite direction than expected, see Figure 1). Third, the interaction between expressed parental anxiety and infant BI was significant $(-2 \log =1243.75, \quad A I C=1293.75, \quad B I C=1397.84)$ confirming an increased role of expressed parental anxiety for high BI children. The three-way interaction between parent gender, expressed parental anxiety and infant BI was also tested in an additional step and found to be significant $(\beta=-19, S E=.09$, $p=.026)$. However, further inspection of the plot revealed significant leverage caused by four mothers with expressed anxiety scores $2.5 S D$ above the mean in five observations. Once these observations were removed, the three-way interaction between parent gender, expressed parental anxiety and infant $\mathrm{BI}$ was no longer significant, $(\beta=-14, \mathrm{SE}=.09, \mathrm{p}=$ .113); therefore, the three-way interaction was not included in the final model. The two-way interactions between expressed parental anxiety and infant BI and between parent gender and parental encouragement remained significant after the removal of the leverage. The interaction between infant BI and parental encouragement was only marginally significant $(\beta=.09, S E=.05, p=.060)$ and did not significantly improve the fit $(-2 \log =1240.28$, $A I C=1292.28, B I C=1400.52)$ and therefore was not included in the final model. The final model (see Table 4) explained $10.11 \%$ of the variance in infant avoidance.

The main effects in the final avoidance model (see Table 4) revealed that infants showed significantly 
less avoidance in the dinosaur SR task $(M=1.61$, $S D=.63)$ than in the stranger $\mathrm{SR}$ task $(M=1.79$, $S D=.57)$. Lifetime parental anxiety diagnoses did not significantly predict infant avoidance. Infant avoidance did not significantly differ between boys and girls.

The interactions between parent gender and parental encouragement and between expressed parental anxiety and infant BI were significant in the final avoidance model. To investigate how the association between parental encouragement and infant avoidance differed between parents, we first inspected the confidence bands [Preacher et al. (2006), see Figure 1A] representing continuously plotted confidence intervals for the simple outcome slope across levels of parental encouragement. The inspection of the confidence intervals revealed that paternal encouragement had its largest impact on infant avoidance with the most and the least encouraging parents. Next, we plotted the association between parental encouragement and infant avoidance in separate lines for mothers and fathers. Confidence intervals cannot be computed for dichotomous variables, such as parent gender; therefore, we based our interpretations on the visual inspection of the interaction plot (shown in Figure 1B). There was a positive association between parental encouragement and infant avoidance for fathers, with higher levels of encouragement by the father being associated with higher levels of avoidance by the infant. Maternal encouragement, however, had a negligible relation with infant avoidance (see Figure 1B).

To investigate how the association between expressed parental anxiety and infant avoidance differed across BI levels, we first inspected the confidence bands [Preacher et al. (2006), see Figure 2A] representing continuously plotted confidence intervals for the simple outcome slope across levels of infant BI. The inspection of the confidence intervals revealed that there was a significant association between expressed parental anxiety and infant avoidance for moderate-to-high BI infants. Next, we inspected the confidence bands (see Figure 2B) representing continuously plotted confidence intervals for the simple outcome slope across levels of expressed parental anxiety. The inspection of the confidence intervals revealed that there was a positive association between expressed parental anxiety and infant avoidance for moderate-to-high levels of expressed parental anxiety. Finally, we plotted the association between expressed parental anxiety and infant avoidance as a function of infant BI (see Figure 2C). The association between expressed parental anxiety and infant avoidance was positive for moderate and high BI infants.

To investigate the link between lifetime parental anxiety disorders and expressed parental anxiety, we regressed expressed parental anxiety on parental
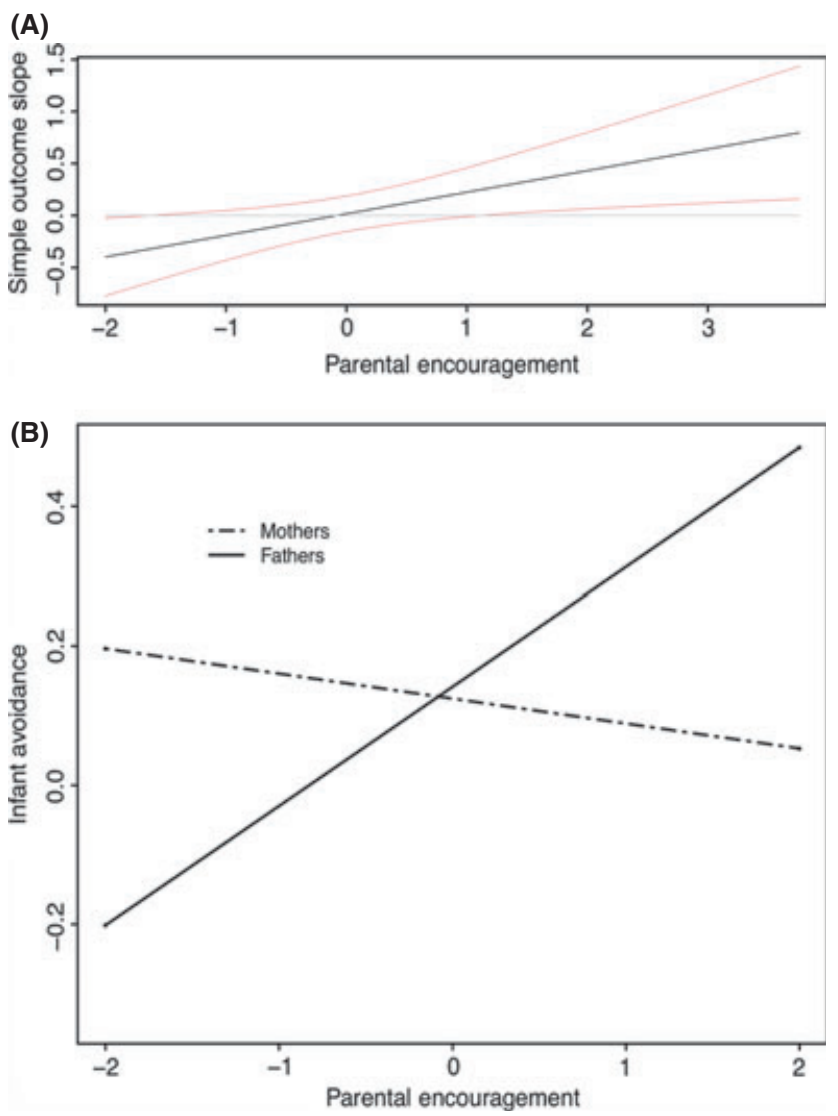

Figure 1 The interaction effect between parent gender and parental encouragement on infant avoidance. (A) The black line is the distribution of simple outcome slope (on the $y$-axis) continuously plotted across levels of parental encouragement (standardized scores on the $x$-axis). The confidence bands (the area bounded by curves) represent the $95 \%$ confidence intervals. When the confidence interval does not include $y=0$ for a given level of the parental encouragement, the effect is interpreted as being significant at that level (i.e. $p \leq .05$ ). The light horizontal line at $y=0$ shows the zero point for the slope (i.e. no association). The association between parental encouragement and infant avoidance was significant among the most encouraging parents $(z \geq 1.11)$ and the least encouraging parents $(z \leq-1.6)$. (B) The interaction between parent gender and parental encouragement. Lines represent the association between parental encouragement (standardized scores on the $x$-axis) and infant avoidance (standardized scores on the $y$-axis) with separate lines for mothers and fathers. The association between parental encouragement and infant avoidance was positive only for fathers, whereas maternal encouragement had a negligible relation with infant avoidance

lifetime anxiety disorders $\quad(-2 \log =1124.37$, $A I C=1142.37, B I C=1179.88)$. None of the interactions between task and parental lifetime anxiety disorders were significant, or improved the model. Parents with lifetime social anxiety $(\beta=.27$, $S E=.14, p=.053)$ and parents with lifetime comorbid social and other anxiety disorders $(\beta=.27$, $S E=.14, p=.054)$ tended to express more anxiety than parents without anxiety disorders. Expressed parental anxiety did not differ between parents with other anxiety disorders and parents without anxiety disorders. Mothers and fathers expressed similar levels of anxiety, and both parents expressed more 
(A)

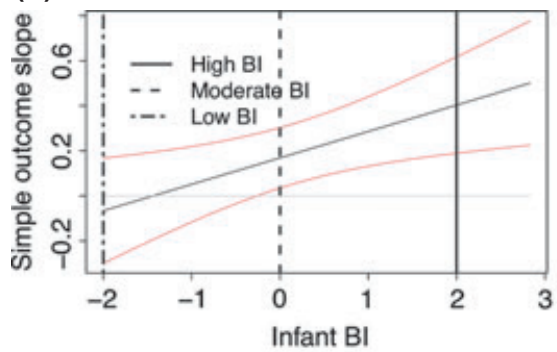

(B)

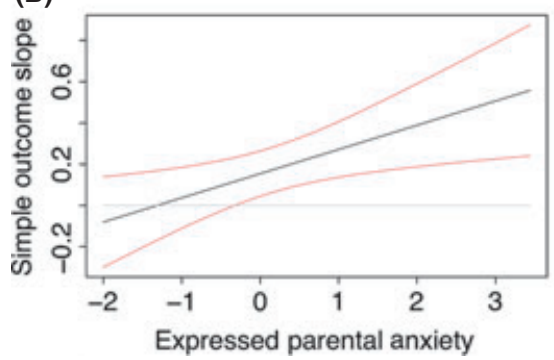

(C)

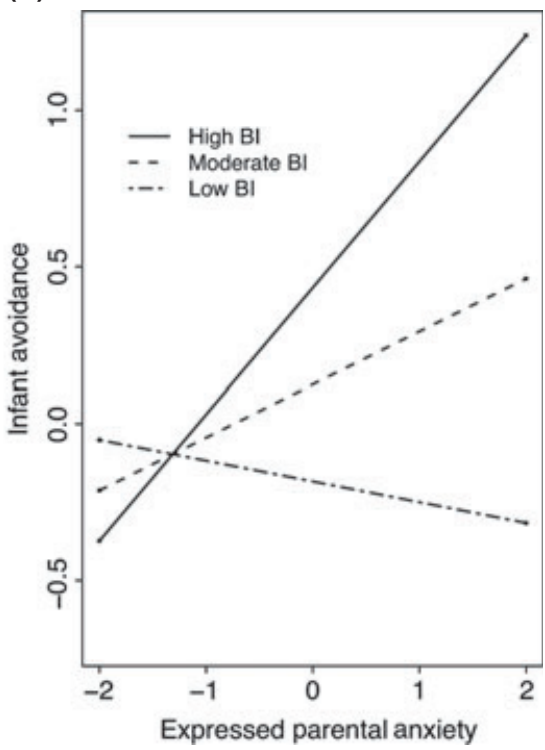

Figure 2. The interaction effect between infant $\mathrm{BI}$ and expressed parental anxiety on infant avoidance. (A) The black line is the distribution of simple outcome slope (on the $y$-axis) continuously plotted across levels of infant behavioural inhibition (BI; standardized scores on the $x$-axis). The confidence bands (the area bounded by curves) represent the $95 \%$ confidence intervals. When the confidence interval does not include $y=0$ for a given level of infant $\mathrm{BI}$, the effect is interpreted as being significant at that level (i.e. $p \leq .05$ ). The light horizontal line at $y=0$ shows the zero point for the slope (i.e. no association). The three vertical lines represent the mean slopes of infants with low, moderate and high levels of infant BI. Moderate, low and high levels of infant BI were determined as mean, $2 S D$ below and $2 S D$ above the mean respectively. There was a significant positive association between infant $\mathrm{BI}$ and infant avoidance among infants with moderate-to-high levels of BI $(z \geq-.03)$. (B) The black line is the distribution of the simple outcome slope (on the $y$-axis) continuously plotted across levels of expressed parental anxiety (standardized scores on the $x$-axis). The confidence bands (the area bounded by curves) represent the $95 \%$ confidence intervals. When the confidence interval does not include $y=0$ for a given level of expressed parental anxiety, the effect is interpreted as being significant at that level (i.e. $p \leq .05$ ). The light horizontal line at $y=0$ shows the zero point for the slope (i.e. no association). Moderate, low and high levels of expressed parental anxiety were determined as mean, $2 S D$ below and $2 S D$ above the mean respectively. Expressed parental anxiety significantly predicted infant avoidance for parents with moderate-to-high levels of expressed parental anxiety $(z \geq-.03)$. (C) The interaction effect between expressed parental anxiety and infant BI. Lines represent the association between expressed parental anxiety (standardized scores on the $x$-axis) and infant avoidance (standardized scores on the $y$-axis) with separate lines for the high, moderate and low BI infants. As indicated in (A), moderate, low and high levels of infant BI were determined as mean, $2 S D$ below and $2 S D$ above the mean respectively. There was a significant positive association between expressed parental anxiety and infant avoidance for moderate-to-high $\mathrm{BI}$ infants

anxiety $(\beta=-.85, S E=.05, p<.001)$ in the stranger task $(M=2.34, S D=.49)$ than in the dinosaur task $(M=1.87, S D=.52)$.

To investigate the possibility that the lack of significant associations between parental anxiety disorders and infant fear/avoidance was caused by the inclusion of both current and lifetime anxiety disorders, we conducted the same analyses only with the current anxiety diagnoses of the parents. The conclusions were similar except that infants of parents with other types of anxiety disorders tended to be more avoidant during SR tasks $(\beta=.32, S E=.16$, $p=.053)$ than infants of parents without current anxiety disorders. Another difference emerged with respect to the link between parental anxiety diagnoses and expressed parental anxiety: parents with a current social anxiety disorder expressed significantly more anxiety than parents without anxiety disorders $(\beta=.36, \quad S E=.16, \quad p=.024)$, whereas parents with current other anxiety disorders and current comorbid social and other anxiety disorders expressed similar levels of anxiety as the parents without anxiety disorders.

\section{Discussion}

The central finding of this study is that expressed parental anxiety interacted with infant BI to significantly predict 12-month-old infants' avoidance in response to a novel stranger and toy. The effect of expressed parental anxiety on infant avoidance was significant for infants with moderate-to-high BI and for parents with moderate-to-high levels of expressed anxiety, whereas the association was not significant for infants with low BI and parents with low levels of expressed anxiety. The findings support the idea that infants may be differentially susceptible to anxious parental rearing based on their level of BI (Belsky \& Pluess, 2009; Nigg, 2006). This study replicates previous findings showing increased vulnerability of high BI infants to the effects of parental anxiety during SR (De Rosnay et al., 2006; Murray et al., 2008).

On the other hand, no evidence was found in favour of increased susceptibility of high BI infants to the beneficial effects of parental encouragement. This may be partially due to the instructions of encouragement in the dinosaur SR task (see below). 
The second major finding of the study is that the link between infant avoidance and expressed parental anxiety did not significantly differ between mothers and fathers, indicating that fathers may be as important as mothers in the transmission of anxiety via SR. Although the analyses initially indicated a stronger link between infant avoidance and expressed parental anxiety for mothers, the interaction was no longer significant once the differences between the parents on parental encouragement were controlled for in the model.

Contrary to the previous evidence indicating an association between more maternal encouragement and less infant avoidance (Murray et al., 2008), maternal encouragement had a negligible relation with infant avoidance in this study, whereas more paternal encouragement was associated with more infant avoidance. The raw correlations between parental encouragement and expressed anxiety revealed that more encouragement in fathers, but not in mothers, was associated with less expressed parental anxiety in dinosaur SR task (see Table 3). It is important to note that the instruction to actively encourage the infant in the last minute of the dinosaur SR task may have increased the occurrence of parental encouragement while leaving the expressions of parental anxiety intact, as some parents (especially mothers, who tend to be more sensitive to infant signals; Barnett, Deng, Mills-Koonce, Willoughby, \& Cox, 2008) may prefer not to actively encourage their infant to approach the dinosaur. This may explain the lack of associations between maternal encouragement and expressed maternal anxiety. In turn, consistent with previous evidence (Barnett et al., 2008), highly encouraging fathers may be encouraging their infant in a less sensitive way as compared with mothers during SR situations, reversing the positive effects of encouragement, triggering further avoidance of the dinosaur. Alternatively, fathers may simply be encouraging more when their infant becomes avoidant in the situation. Additional research is needed to discover the mechanisms that account for the positive link found between encouragement from fathers and infant avoidance. The present findings reveal that parental encouragement may not always help infants to decrease their avoidance in face of ambiguous stimuli.

In contrast with infant avoidance, infant fear was only predicted by infant BI. The more behaviourally inhibited infants were, the more fear they showed during SR, pointing to a moderate effect of this temperamental predisposition on infant fear. This finding may provide support for the role of the early temperamental trait of BI in the acquisition/learning of fear (Rosenbaum et al. 1993). Expressed parental anxiety was not significantly associated with infant fear, indicating that infant's own fearful disposition, and not his/her parents' anxiety was important for fear expression in the SR tasks. It is important to note that observed infant BI was considered to be a measure of 'trait' fear in this study, whereas infant fear in SR situations was considered to reflect the infant's 'state' reaction to one social and one nonsocial novelty, in the presence of parental feedback. However, in light of the definitional and methodological overlap between fear and the temperamental predisposition of anxiety (Fox et al., 2005; Rothbart, 2007), one may suggest that the procedures of infant BI and infant fear were similar, leading to the conclusion that the present model on infant fear reflected an absence of significant parental effects (i.e. expressed parental anxiety) on infant fear reactions, which were mainly consistent from BI to SR situations. As infant BI was measured in a range of situations in this study, (i.e. at home and in two different lab visits), using 11 well-known standardized tasks, and in the absence of parental feedback, we consider our BI measure distinct enough from the fear measure to warrant the present conclusions about state and trait fear.

The third major finding of the study is that lifetime and current parental anxiety disorders were not significantly related to infant fear or avoidance during SR tasks, with the exception of a current diagnosis of other types of anxiety. It is difficult to understand why infants of parents with other (nonsocial) anxiety disorders, but not those with social or with comorbid social and other anxiety disorders were more avoidant than infants of parents without anxiety disorder. Taken together, the findings do not support the diagnosis-specificity hypothesis for the early transmission of anxiety. Although the lack of association between parental lifetime anxiety diagnoses and infant avoidance seems in contrast with Murray et al. (2008) who reported more avoidance in infants of mothers with social anxiety disorder, it should be kept in mind that the parental anxiety diagnoses were analysed in the present models simultaneously with expressed parental anxiety. It is important to note that parental anxiety diagnoses, measured via the ADIS were considered to be a measure of parental 'trait' anxiety in this study, whereas observations of expressed parental anxiety in SR situations were considered to reflect the parent's 'state' reaction to these situations. The models of infant fear and avoidance in this study could not differentiate between parents with and without anxiety disorders independently from expressed parental anxiety.

With respect to expressed parental anxiety, parents with lifetime and current diagnoses of social anxiety disorders and with lifetime comorbid social and other anxiety disorders did express more anxiety than parents without anxiety disorders during SR situations. The expressions of parental anxiety were thus specific to parents with social anxiety disorder. This makes sense given that being aware that one is being filmed is an experience that is especially likely to trigger social anxiety. However, parents with a 
lifetime or current diagnosis of other anxiety disorders and parents with current diagnoses of comorbid social and other anxiety disorders expressed similar levels of anxiety as parents without anxiety disorders during SR situations.

Several explanations can be offered for the lack of association between infant avoidance and parental anxiety disorders. Parents may be able to mask their anxiety disorders when helping their infants approach a novel stimulus, either because the anxiety disorder is not triggered enough in the SR tasks, or because some parents with anxiety disorders are able to hide their anxiety from their infant. Expressed parental anxiety predicted infant avoidance over and above the effects of lifetime anxiety disorders, implying that the way a parent acts in response to novel stimuli in the presence of the infant matters more than his/her predisposition towards an anxiety disorder.

The fourth major finding of this study is that neither the expressions of parental anxiety, nor their effect on infant behaviour were found to be contextspecific. Parents' behaviour influenced infants' behaviour similarly in social and nonsocial SR contexts. The association between expressed parental anxiety and parental anxiety disorders did not significantly differ between SR contexts, providing evidence against context-specificity in the transmission of anxiety.

Infants were less fearful and more avoidant in the stranger SR task than in the dinosaur SR task. No gender difference was detected between the boys' and girls' expressions of fear and avoidance and/or between the mothers' and fathers' expressions of anxiety, suggesting that the transmission of anxiety may be occurring similarly from mothers and fathers to girls and boys. The link between BI and infant fear/avoidance did not significantly differ between mother and father visits, implying a similar influence of infant temperamental characteristics on infant reactions with mothers and fathers.

The number of times the infant looked at the parent was not significantly associated with infant fear or avoidance in the SR situations in the multilevel models, whereas the raw correlations between BI and looks at the parent indicated a positive association in the stranger SR task of the mother's visit. Although the number of looks is an indicator of active visual information seeking, it is certainly not the only valid proxy in the naturalistic setting of the present SR tasks. Infants were provided with multimodal (visual and auditory) signs of expressed parental anxiety. Previous studies comparing visual and auditory modalities in SR situations (Mumme, Fernald, \& Herrera, 1996) suggest that, in fact, auditory (vs. visual) information may be a stronger channel for fearful expressions during SR situations. In addition, multimodal input can enhance early emotional information processing (Flom \& Bahrick, 2007).

Findings of this study should be interpreted in the light of some limitations. First, the study focused on the predictive value of expressed parental anxiety on infant fear and avoidance and did not consider the opposite direction of effects (i.e. infant fear/avoidance on expressed parental anxiety). Parents' expressed anxiety during SR situations may partly result from the simultaneous fear/avoidance of the infant in that situation. Second, the reliability of the infant avoidance was slightly lower than conventionally acceptable levels for observational measures. This may partly stem from the structure of the stranger SR task where the infants were restrained in a high chair. As a result, the avoidant behaviour, which is defined as increasing the distance between self and the ambiguous stimulus was manifest in less obvious ways, possibly resulting in lower levels of the interobserver agreement. Third, the study had a singleage nonexperimental design, therefore, the present conclusions preclude any prospective or causal inferences about the effect of expressed parental anxiety on infant fear or avoidance. It is important to follow up the present sample to investigate the prospective effects of expressed parental anxiety on infant behaviour in a SR paradigm.

\section{Conclusions}

This study supports the notion that infants who show a temperamental disposition for anxiety learn from maternal and paternal anxious signals during confrontations with novelty and become avoidant towards novel stimuli when their parents express anxiety. Consistent with previous evidence, the findings suggest that moderate and high levels of BI constitute a temperamental vulnerability for the effects of expressed parental anxiety.

This was the first study to our knowledge comparing fathers' and mothers' effects on their infants in SR tasks. The association between infant avoidance and expressed parental anxiety was not significantly different for mothers and fathers, pointing to an equally important role of fathers at this young age. Contrary with the previous evidence showing negative associations between maternal encouragement and infant avoidance, the association was only significant for fathers in this study and it was positive, that is infants of highly encouraging fathers showed higher levels of avoidance.

This study did not find evidence in favour of diagnosis- or context-specificity of the intergenerational transmission of anxiety via SR. The association between expressed parental anxiety and infant fear/avoidance was found to be independent of the presence of anxiety disorders in parents, and similar in social versus nonsocial SR contexts. 


\section{Acknowledgements}

The contribution of Evin Aktar was supported by the research priority program 'Brain and Cognition'. The contributions of Mirjana Majdandžić, Wieke de Vente and Susan Bögels were supported by the Innovation Research Vici NWO grant, number 453-09-001, awarded to Susan Bögels. Authors are grateful to Prof. Lynne Murray, Prof. Peter Cooper and Dr. Cathy Creswell from the University of Reading for their cooperation on the SR tasks, and to Dr. Dorothy
Mandell and to Prof. Frans Oort from the University of Amsterdam for their support on the statistical analyses.

\section{Correspondence}

Evin Aktar, Cognitive Science Center Amsterdam, University of Amsterdam, Nieuwe Prinsengracht 130, 1018 VZ, Amsterdam, The Netherlands; Email: e.aktar@ uva.nl

\section{Key points}

- Previous evidence revealed that high $\mathrm{BI}$ infants of mothers with social anxiety disorder learn from maternal anxious signals via SR and become progressively avoidant towards strangers. This study extended previous research by comparing SR effects from fathers and mothers, by investigating specificity of SR processes in a social and a nonsocial situation, and by studying anxiety subtype specificity of SR via the inclusion of parents lifetime social anxiety and/or other anxiety disorders.

- The link between infant avoidance and expressed parental anxiety did not significantly differ between mothers and fathers, pointing to an equally important role of fathers in the transmission of anxiety.

- Contrary with the previous evidence indicating an association between more maternal encouragement and less infant avoidance (Murray et al., 2008), maternal encouragement had a negligible relation with infant avoidance in this study, whereas more paternal encouragement was associated with more infant avoidance.

- Expressed parental anxiety during SR (but not lifetime parental anxiety disorders) interacted with infant BI to predict infant avoidance. The effect of expressed parental anxiety was significant for moderate-to-high $\mathrm{BI}$ infants and for parents with moderate-to-high levels of expressed anxiety.

- Infant fear was predicted by infant BI and not by expressed parental anxiety.

- The association between infant fear/avoidance and expressed anxiety did not significantly differ across SR contexts.

\section{References}

Barnett, M.A., Deng, M., Mills-Koonce, W.R., Willoughby, M., \& Cox, M. (2008). Interdependence of parenting of mothers and fathers of infants. Journal of Family Psychology, 22, 561-573.

Belsky, J., \& Pluess, M. (2009). Beyond diathesis stress: Differential susceptibility to environmental influences. Psychological Bulletin, 135, 885-908.

Biederman, J., Rosenbaum, J.F., Chaloff, J., \& Kagan, J. (1995). Behavioural inhibition as a risk factor for anxiety disorders. In J.S. March (ed.), Anxiety disorders in children and adolescents (pp. 61-81). New York: Guilford Press.

Bögels, S.M., \& Brechman-Toussaint, M.L. (2006). Family issues in child anxiety: Attachment, family functioning, parental rearing and beliefs. Clinical Psychology Review, 26, 834-856.

Bögels, S.M., \& Perotti, E.C. (2011). Does father know best? A formal model of the paternal influence on childhood social anxiety. Journal of Child and Family Studies, 20, 171-181.

Bögels, S.M., \& Phares, V. (2008). Fathers' role in the etiology, prevention and treatment of child anxiety: A review and a new model. Clinical Psychology Review, 28, 539-558.

Bradshaw, D.L., Goldsmith, H.H., \& Campos, J.J. (1987). Attachment, temperament, and social referencing: Interrelationships among three domains of infant affective behaviour. Infant Behaviour and Development, 10, 223231.

Calkins, S.D., Fox, N.A., \& Marshall, T.R. (1996). Behavioural and physiological antecedents of inhibited and uninhibited behaviour. Child Development, 67, 523-540.

Carpenter, M., Nagell, K., Tomasello, M., Butterworth, G., \& Moore, C. (1998). Social cognition, joint attention, and communicative competence from 9 to 15 months of age. Monographs of the Society for Research in Child Development, 63, 1-176.

De Rosnay, M., Cooper, P.J., Tsigaras, N., \& Murray, L. (2006). Transmission of social anxiety from mother to infant: An experimental study using a social referencing paradigm. Behaviour Research and Therapy, 44, 1165-1175.

Di Nardo, P.A., Brown, T.A., \& Barlow, D.H. (1994). Anxiety disorders interview schedule for DSM-IV: Lifetime version (ADIS-IV-L). San Antonio, TX: Psychological Corporation.

Dubi, K., Rapee, R.M., Emerton, J.L., \& Schniering, C.A. (2008). Maternal modeling and the acquisition of fear and avoidance in toddlers: Influence of stimulus preparedness and child temperament. Journal of abnormal child psychology, 36, 499-512.

Eley, T.C. (2001). Contributions of behavioural genetics research: Quantifiying genetic, shared environmental and non-shared environmental influences. In M.W. Vasey \& M.R. Dadds (Eds.). The developmental psychopathology of anxiety (pp. 45-59). New York: Oxford University Press.

Feinman, B. (1982). Social referencing in infancy. MerrillPalmer Quaterly, 28, 445-470.

Fisak, B. Jr, \& Grills-Taquechel, E.A. (2007). Parental modelling, reinforcement, and information transfer: Risk factors in the development of child anxiety? Clinical Child and Family Psychology, 10, 213-231.

Flom, R., \& Bahrick, L.E. (2007). The development of infant discrimination of affect in multimodal and unimodal stimulation: The role of intersensory redundancy. Developmental psychology, 43, 238-252.

Fox, N.A., Henderson, H.A., Marshall, P.J., Nichols, K.E., \& Ghera, M.M. (2005). Behavioural inhibition: Linking biology and behaviour within a developmental framework. Annual Review of Psychology, 56, 235-262. 
Fox, N.A., Henderson, H.A., Rubin, K.H., Calkins, S.D., \& Schmidt, L.A. (2001). Continuity and discontinuity of behavioral inhibition and exuberance: Psychophysiological and behavioral influences across the first four years of life. Child Development, 72, 1-21.

Gerull, F.C., \& Rapee, R.M. (2002). Mother knows best: Effects of maternal modelling on the acquisition of fear and avoidance behaviour in toddlers. Behaviour Research and Therapy, 40, 279-287.

Goldsmith, H.H., \& Rothbart, M.K. (1996). The laboratory temperament assessment battery, locomotor version (manual), Technical Manual, Department of psychology, University of Wisconsin, Madison, WI, USA.

Hettema, J.M., Neale, M.C., \& Kendler, K.S. (2001). A review and meta-analysis of the genetic epidemiology of anxiety disorders. American Journal of Psychiatry, 158, 1568-1578.

Ingram, R.E., \& Luxton, D. (2005). Vulnerability-stress models. In B.L. Hankin \& J.R.Z. Abela (Eds.), Development of psychopathology: A vulnerability-stress perspective (pp. 3246). New York: Sage.

Kagan, J., \& Snidman, N. (1999). Early childhood predictors of adult anxiety disorders. Society of Biological Psychiatry, 46, $1536-1541$.

Kashani, J.H., \& Orvaschel, H. (1990). A community study of anxiety in children and adolescents. American Journal of Psychiatry, 147, 313-318.

Kessler, R.C., Chiu, W.T., Demler, O., \& Walters, E.E. (2005) Prevalence, severity, and comorbidity of 12-month DSM-IV disorders in the national comorbidity survey replication. Archives of General Psychiatry, 62, 617-627.

Kochanska, G., Coy, K.C., Tjebkes, T.L., \& Husarek, S.J (1998). Individual differences in emotionality in infancy. Child Development, 69, 375-390.

Mumme, D.L., Fernald, A., \& Herrera, C. (1996). Infants' responses to facial and vocal emotional signals in a social referencing paradigm. Child Development, 67, 3219-3237.

Muris, P., Steerneman, P., Merckelbach, H., \& Meesters, C. (1996). The role of parental fearfulness and modeling in children's fear. Behaviour Research and Therapy, 34, 265268.
Murray, L., Creswell, C., \& Cooper, P.J. (2009). The development of anxiety disorders in childhood: An integrative review. Psychological Medicine, 39, 1413-1423.

Murray, L., De Rosnay, M., Pearson, J., Bergeron, C., Schofield, E., Royal-Lawson, M., \& Cooper, P.J. (2008). Intergenerational transmission of social anxiety: The role of social referencing processes in infancy. Child Development, 79, $1049-1064$.

Nigg, J.T. (2006). Temperament and developmental psychopathology. Journal of Child Psychology and Psychiatry, 47, 395-422.

Preacher, K.J., Curran, P.J., \& Bauer, D.J. (2006). Computational tools for probing interaction effects in multiple linear regression, multilevel modeling, and latent curve analysis. Journal of Educational and Behavioural Statistics, 31, 437448.

Rapee, R.M. (2001). The development of generalized anxiety. In M.R. Dadds \& M.W. Vasey (Eds.), The developmental psychopathology of anxiety (pp. 481-503). London: Oxford University Press.

Rosenbaum, J.F., Biederman, J., Bolduc-Murphy, E.A., Faraone, S.V., Chaloff, J., Hirshfeld, D.R., \& Kagan, J. (1993). Behavioural inhibition in childhood: A risk factor for anxiety disorders. Harvard Review of Psychiatry, 1, 2-16.

Rothbart, M.K. (1988). Temperament and the development of inhibited approach. Child Development, 59, 1241-1250.

Rothbart, M.K. (2007). Temperament, development, and personality. Current Directions in Psychological Science, 16, 207-213.

Rubin, K.H., Coplan, R.J., \& Bowker, J.C. (2009). Social withdrawal in childhood. Annual Review of Psychology, 60, 141-171.

Sroufe, L.A. (1977). Wariness of strangers and the study of infant development. Child Development, 48, 731-746.

Zuckerman, M. (1999). Vulnerability to psychopathology: A biosocial model. Washington, DC: American Psychological Association.

Accepted for publication: 1 June 2012

Published online: 27 August 2012 